\title{
A Basic Study of Auction-based Planning and Scheduling for Cell Manufacturing
}

\author{
Susumu Fujii ${ }^{1}$, Toshiya Kaihara ${ }^{2}$, Kentaro Sashio ${ }^{2}$, Hiroko Yokose ${ }^{2}$, \\ Masasi Kurahashi ${ }^{3}$ and Nobuhiro Hayashi ${ }^{3}$ \\ 1. Sophia Univeristy, Department of Mechanical Engineering \\ 7-1, Kioi-cho, Chiyoda-ku, Tokyo, 102-8554 Japan \\ 2. Kobe University, Dept. of Computer and Systems Engineering \\ 1-1, Rokkodai, Nada, Kobe 657-8501 Japan \\ 3. Omron Corporation, Industrial Automation Company, \\ Production Management \&System Engineering Group, \\ 2-2-1, Nishikusatsu, Kusatsu, 525-0035 Japan
}

\begin{abstract}
Cell manufacturing is widely introduced to cope with the dynamically changing market demands. This study considers a scheduling method for a cell assembly system for the products that are characterized by the type and specification. A major set up is required at a cell for the change of types and a minor one for the change of specification within the same types. The scheduling will be made in three folds. Firstly, the scheduling for the orders with due dates of the day is made before the processing of the day starts. If cells have slack times for the production of the orders of the day, express orders to be completed within the day will be accepted in a prespecified time period or until no cells become available for the production. If slack times still exist, advanced production of orders with future due dates will be considered. Auction-based algorithms are proposed for the scheduling and their effectiveness is investigated by simulation studies.
\end{abstract}

\section{Introduction}

Cell manufacturing is widely introduced in assembly shops especially in the assembly of electronic devices enabling to keep the agility and the flexibility of the system and to respond the dynamically changing market demands [1]. A cell manufacturing system is constituted of several cells each of which may be also constituted of some stations. One product is assembled in a cell moving from station to station devised with appropriate tools and/or equipments. The product kinds can be changed relatively easily by changing the setups at each station. To attain the high performance out of the system, it is important not only to provide hardware with

Please use the following format when citing this chapter:

Fujii, S., Kaihara T., Sahio, K. Yokose, H., Kurahashi, M. and Hayashi, N., 2008, in IFIP International Federation for Information Processing, Volume 257, Lean Business Systems and Beyond, Tomasz Koch, ed.; (Boston: Springer), pp. 3-10. 
high flexibility but also to have a planning and scheduling systems which is also easy to cope with the dynamic changes of the demands in the market.

In this study, an assembly system for electronic devices is considered to be redesigned into cell manufacturing. The products are largely classified into kinds, each of which is further classified into types. The objective system needs to process a large number of orders per day. Most of orders are ordinary orders received before the day starts, but some express orders requiring the shipping in the same day are also received and accepted if possible. To improve the customers' satisfaction, the primary objective of the new system is set to ship out all ordinary orders without any delay with a limited number of cells and then to accept express orders as many as possible as the secondary objective. To cope with these objectives, the planning and scheduling procedures based on the auction are proposed for agile manufacturing.

This paper describes the outline of the objective manufacturing system and the frame of the auction based planning and scheduling procedures. Then the basic design of the planning and scheduling procedures is discussed using a numerical example. The effectiveness of the proposed system will be also demonstrated in conjunction with the designing procedure of the frame.

\section{Outline of Objective Manufacturing System}

The conceptual configuration of the cell manufacturing system in this study is shown in Fig. 1. The system has $\mathrm{N}$ cells and each cell is constituted by some stations, six stations in this study. The products are largely classified into some kinds and products in a same kind are further classified into some types. All products are processed at each station for a pre-determined fixed cycle time regardless to the kinds and types, 15 seconds in this study. For the change in the product kind, each cell needs to stop for a large setup change, 10 minutes in this study, and for the change in the type within the same kind, the setup at one station is made station to station, and one cycle time at a station, 15 seconds in this study, will be required for the setup change.

The orders usually received in advance specifying the product kind and type, the number of products (order size) and the due date for delivery. These orders are called ordinary orders in this study. The shipping date is estimated based on the distance and the due date for delivery and the shipping date becomes the due date at the factory. In this study an order whose completion time is before T, 17:00 in this study, can be shipped out by the last truck scheduled on the day and the order is considered to be in time or without delay. Because of a short processing time of the product in this study, most of the ordinary orders are scheduled for manufacturing on the day of shipping. Such ordinary orders whose shipping date is same as the manufacturing date are called today's order hereafter. In addition, special orders, express orders in this study, will be accepted by the sales department and are also expected to be completed for the shipping of the day. Since today's orders have the first priority, express orders will only be accepted up to the total capacity of the system. 


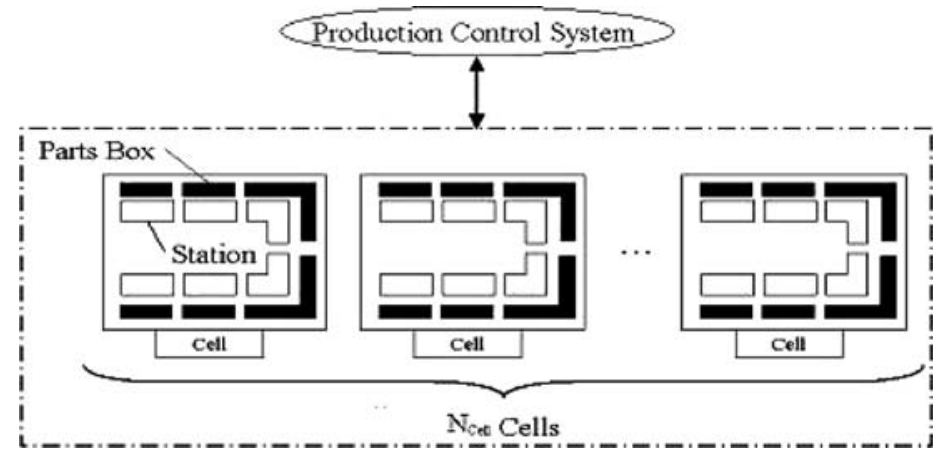

Fig. 1. Conceptual Configuration of Objective Cell System

\section{Frame of Daily Planning and Scheduling}

For the customers' satisfaction, the primary objective of the system is to complete all ordinary orders without any delay and the secondary one is to accept the express orders as many as possible under the constraint of given number of cells and the regular working hours. Every day, by T0 before the operation of the day starts, say 9:00, the ordinary today's orders are to be assigned to cells. This is a static scheduling of a given set of orders. At T0, all cells starts their operation based on the schedule of today's orders. The express orders received by the sales department are assigned to some cells based on some scheduling rules dynamically. If the system has some slacks even after accepting all express orders in addition to the today's orders, the system can process the future orders, whose shipping dates are tomorrow or later, in advance.

A scheduling system pursuing the optimum may be developed to produce a schedule which minimizes the setup times for today's orders in advance and can inform the available time for express orders to the sales department. It will however take a long time for scheduling a large number of orders and will not be practical to re-run the scheduling system every time the express orders are received to obtain the optimum schedule.

To solve these problems, a new auction based planning and scheduling procedures are proposed taking the advantage of cell manufacturing system into consideration. The procedures for today's, express and future orders are separately developed and then integrated as briefly described below and shown in Fig. 2. The working hour of one day starts at $\mathrm{T} 0$ and ends at $\mathrm{T}$. The orders completed by $\mathrm{T}$ can be shipped out in this day.

Scheduling of today's orders (Static Scheduling): At the beginning of the day, T0, today's orders are scheduled by the auction. This is called planning auction in this study.

Scheduling of express orders (Dynamic Scheduling): Express orders will be received after the daily operation starts. As far as any cell has a slack to process 
them, the express order will be basically accepted. The auction is made to determine when the order is processed on which cell.

Scheduling of advanced future orders (Dynamic Scheduling): Suppose that cells have slack times even after processing all accepted express orders. Then it will be appropriate to process future orders in advance to attain a high utilization. After T1, each cell which becomes free starts to seek future orders to process by auction.

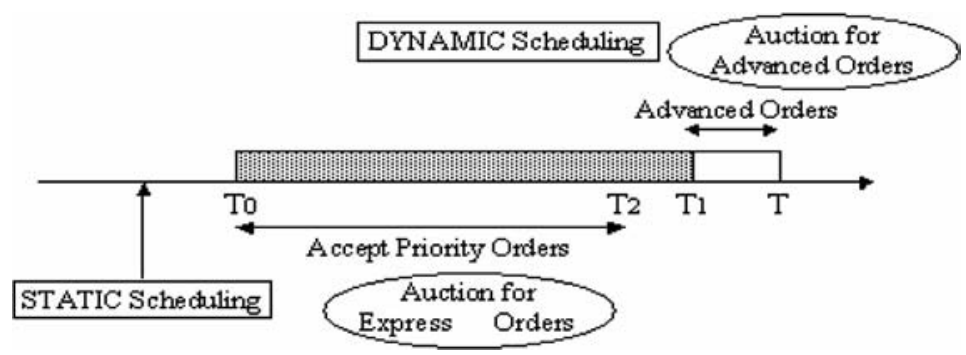

Fig. 2. Time Chart for Daily Planning and Scheduling

\section{Basic Design of Objective Manufacturing System}

To develop an efficient scheduling system, it is necessary to customize the basic policy and parameters to the objective manufacturing system. In this study, the scheduling system is developed for the manufacturing system with 10 cells. The products are classified into 27 kinds and 1035 types in total. The number of products in each order, i. e., order size, ranges from 1 to 100 . Order sizes are grouped into three groups; G1 $=[1-9], \mathrm{G} 2=[10-60]$ and $\mathrm{G} 3=[61-100]$, where the size in one group is assumed uniformly distributed. Orders for each day are generated so that the number of orders with order size in each group will become $\mathrm{P} 1=\{80 \%: 15 \%: 5 \%\}$. For each order the product kind is firstly assigned randomly and then its type are randomly assigned. To investigate the effect of ratio of order sizes in a day, an order pattern $\mathrm{P} 2=\{5 \%, 15 \%, 80 \%\}$ is also considered.

\subsection{Planning Auction for Today's Orders}

The capacity of the system depends on the schedule of orders. In this system a schedule with minimum setup times is the most efficient schedule since the processing times of all products are the same. All today's orders are scheduled before the operation of the day starts and thus the auction for today's orders is named planning auction. The auction procedure is given below, where the item for auction could be one single order or a set of orders to be determined later. 


\section{Planning Auction:}

Step 1: (Co) Set orders into items based on the specified rules.

Item A: A single order

Item B: A set of orders with same kind and type (no setup required)

Item C: A set of Item B with same kind (short setup time required)

Step 2: (Co) Select one item by the specified selection policy. Then the characteristics of the item are announced to cells.

Policy 1: Randomly select Item A

Policy 2: In the decreasing order of total processing time of Item B

Policy 3: In the decreasing order of total processing time of Item $\mathrm{C}$

Step 3: (Cell) Assume to process the item after the last item assigned to the cell. Calculate the setup time and completion time of the item. If the completion time is later than $\mathrm{T}$, the item will be delayed to the shipping time and thus the bid is "not available". If "available", the bids are "setup time" and "present load (=completion time of the last order except the item)".

Step 4: (Co) Select cells with shortest setup time and then select a cell with the lightest present load among the selected cells, i. e., the earliest completion time. If more than one cell remains, select one randomly. The item is awarded to the selected cell. Go to Step 2 if items for auction remain. Otherwise, stop.

\subsection{Efficiency of Planning Auction and System Capacity}

To investigate the efficiency of the planning auction, preliminary experiments are performed for selection policies of items. The results for one set of orders are shown in Fig. 3. From the figure the effectiveness of the policy 3 is obvious, and Policy 3 is adopted in the planning auction in this study.

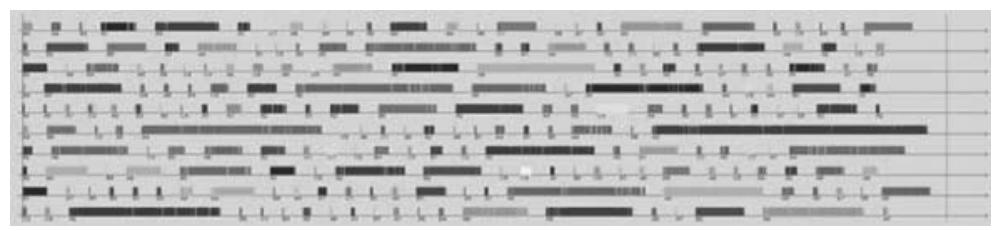

Fig. 3 (a). Policy 1: Item A, Random

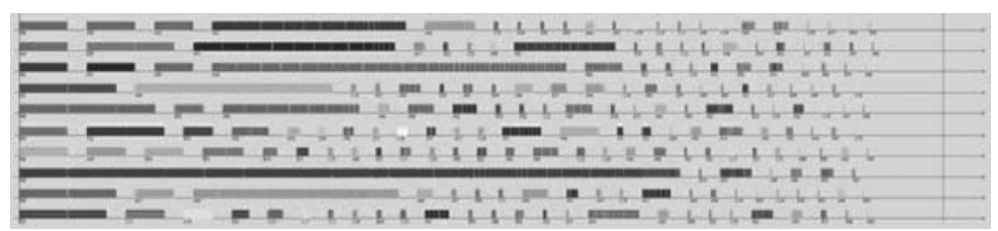

Fig. 3 (b). Policy 2: Item B, Decreasing Order of Processing Time 


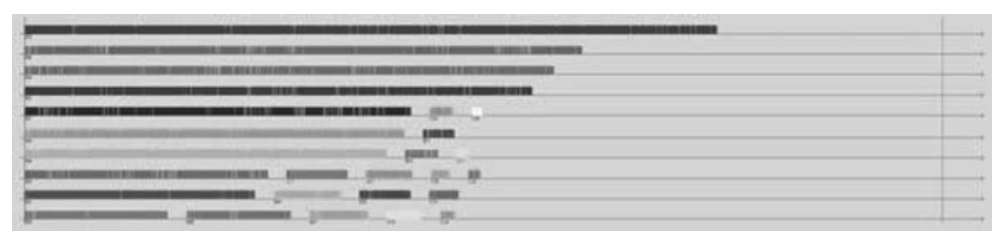

Fig. 3 (c). Policy 3: Item C, Decreasing Order of Processing Time

The capacity of the system can be defined as the maximum number of orders to be processed in one day without any delay. Increasing the number of orders, the system utilization in 8 hours operation and the number of tardy orders which delayed to the shipping are evaluated by running 10 simulations for each number of orders. The averaged results for each number of orders are given in Table 1. Based on the results, the maximum capacity of the system is considered to be around 1300 .

Table 1. System Performance for given Number of Orders

\begin{tabular}{|c|r|r|}
\hline $\begin{array}{c}\text { Number of } \\
\text { Orders }\end{array}$ & $\begin{array}{c}\text { Utilization } \\
(\%)\end{array}$ & $\begin{array}{c}\text { Number of } \\
\text { Delayed Orders }\end{array}$ \\
\hline 1000 & 68.07 & 0 \\
\hline 1100 & 74.41 & 0 \\
\hline 1200 & 79.20 & 6.9 \\
\hline 1300 & 86.89 & 49.5 \\
\hline 1400 & 91.69 & 111.1 \\
\hline 1500 & 92.53 & \\
\hline
\end{tabular}

\subsection{Auction for Express Orders}

The auction for express orders is performed at the time of reception of an express order. This implies that Steps 1 and 2 of the planning auction will be set to Item A and Policy 1, respectively, in the auction for express orders. The bids provided by cells depend on the timing when the express orders are proessed. Two scheduling patterns are considered. If the orders including the express order under consideration cannot be completed in time by any cell, the order will not be accepted.

Pattern 1: Express orders are processed after the order with minimum setup time, provided that the last order on the cell can be completed in time. This means that the schedule is dynamically changed when an express order is accepted. A cell with the lightest load or the earliest completion time of the last order on the cell at the time of auction is selected among those with same minimum setup time. The bids of cells in Step 3 are then "availability (can or cannot complete the all orders in time)", "minimum setup time on the cell" and "present load"

Pattern 2: Express orders are processed after completing the last order assigned to the selected cell. This means the schedule for today's order is fixed. The cell is selected by the lightest load rule. The bids of cells in Step 3 are then "availability" and "completion time of last order assigned by the time auction". 
The effectiveness of the pattern is investigated under the condition that today's orders generated will require 7 hours to process on the average. Typical schedules for these patterns are shown in Fig. 4. It is easily observed that more orders are scheduled when the schedule is dynamically changed inserting the express orders in the schedule of today's orders. Number of express orders generated for 6 hours (= T2- T0) is increased from 80 to 140 and the system utilization and the number of tardy orders are obtained by simulation. The results are shown in Fig. 5 and Pattern 1 shows better performance with 130 to 140 acceptable express orders with less than one tardy orders and higher utilization than that of Pattern 2.

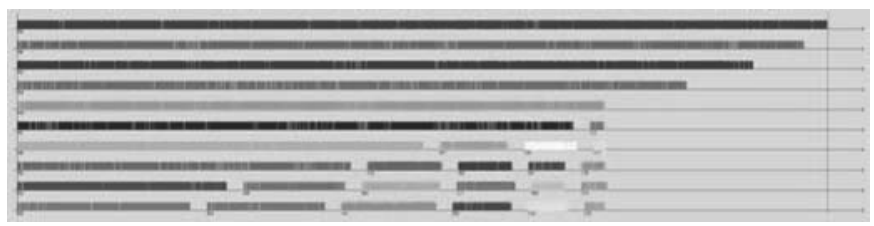

Fig. 4 (a). Schedule of Today's Orders

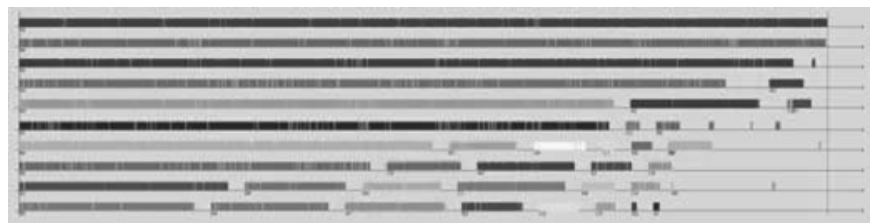

Fig. 4 (b). Schedule of Express Orders with Pattern 1

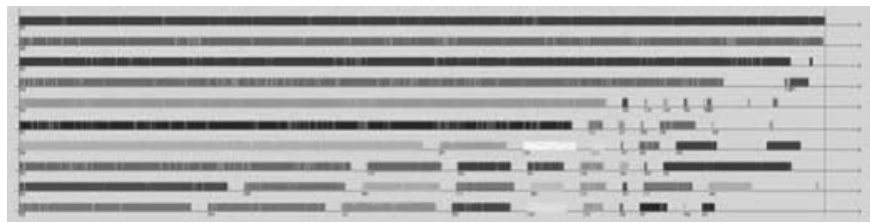

Fig. 4 (c). Schedule of Express orders with Pattern 2

\subsection{Reverse Auction for Advanced Future Orders}

If cells complete all assigned orders before the end time of the day, orders with future shipping dates can be processed. This will be preferable not only to improve the utilization of the system but also to absorb the daily variation of the production volume incurred by the fluctuation in the number of ordinary orders. The auction for such future orders is quite different from the planning auction and auction for express order. The auction is initiated by a cell which has a slack time and thus the cell becomes the coordinator and the future orders are the participants. Since the relation between cell and order are reversed from the planning auction, the auction for future orders is named a reverse auction in this study. 


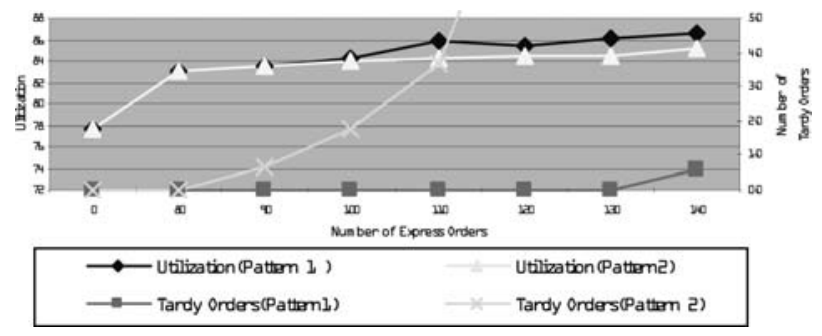

Fig. 5. Effects of Number of Express Orders and Scheduling Patterns

In the reverse auction, the effectiveness is defined to be evaluated by the number of orders or the total production volume manufactured in the slack time. These measures will be affected by the number of orders to be included in the auction. In other words, it will be important to determine the planning horizon. Simulations are run after scheduling the today's orders for 7 working hours, where no express orders are considered. The numbers of future orders processed in the slack time for cases with the planning horizons of one day and two days were 158 and 165, respectively, and the system utilizations were 86.2 and 86.6. Longer planning horizon shows that more future orders can be processed yielding higher utilization. This is explained by the fact that the possibility to have orders of same type or at least of same kind to the last order on the cell will be higher in larger number of orders.

\section{Conclusions}

This paper considers a cell manufacturing system of assembling electronic devises. In addition to a large number of orders to process in a day, express orders are also to be accepted as many as possible. To cope with these requirements, auction based scheduling procedures are proposed as a dynamic and flexible planning and scheduling system. Planning auction is presented for scheduling orders before the daily operation starts. By simulation studies, it was shown that the auction should be made as a group of orders with a same kind in the decreasing order of total groupwise processing time. For express orders, an auction is performed when it is received. High performance will be attained by a scheduling such that orders are processed just after an order of the same type or at lest in the same kind is completed. A reverse auction is proposed for scheduling future orders to be processed in slack times of cells. The experiments showed that higher performance will be attained when longer planning horizon is set for future orders.

To implement these procedures in a system, the period to receive the express orders, the time to start reverse auction and other parameters are to be determined, but they are left for further studies..

\section{References}

1. Cover Story, "Need to avoid vulnerability in Cell Manufacturing", Nikkei Monodukuri, Nikkei BP, July, 2004. (In Japanese). 